\title{
Configuración territorial del turismo en las costas de la isla de Cozumel
}

DOI: 10.22403/UQROOMX/TYP05/23

\section{Claudia Inés Martínez*}

\section{RESUMEN}

El trabajo tiene como objetivo analizar la ocupación territorial del turismo en las costas de la isla de Cozumel, para lo cual se realiza una clasificación jerárquica de los tipos de espacios con uso turístico-recreativo presentes en el litoral cozumeleño. Primero se estudian los antecedentes histórico-geográficos de Cozumel, desde los inicios del turismo hasta la actualidad. Luego se presentan las estrategias metodológicas aplicadas en la investigación, las características territoriales de los espacios costeros con uso turístico y recreativo $y$, finalmente, se examinan patrones territoriales del turismo en la costa de Cozumel.

\begin{tabular}{r|rl} 
Palabras & Cozumel, Costas, recreación, turismo. \\
clave &
\end{tabular}

*Universidad Nacional Autónoma de México, Facultad de Filosofía y Letras, Instituto de Geografía / martinezclaul6@hotmail.com 


\section{Introducción}

Cozumel es una de las islas de México que a partir de los setenta, ha presentado un mayor crecimiento urbano, debido a un fuerte proceso migratorio. Los principales factores que determinaron esta dinámica han sido el aumento de la actividad turística y de la oferta de empleo en este destino.

En los noventa se realizaron inversiones en infraestructura portuaria y a la fecha se cuenta con tres terminales con capacidad para el atraque de seis barcos simultáneamente, que la posicionan como el primer puerto mexicano en recepción de cruceros.

Frente al incremento de la demanda turística, Cozumel ha sufrido grandes transformaciones en su litoral marítimo, sobre todo a partir del desarrollo de dos zonas hoteleras (al noroeste y al suroeste de la isla) y por la construcción de los muelles para el arribo de cruceros.

Esta construcción de equipamiento turístico e infraestructura ha generado un aumento en la oferta turística del destino. Sin embargo, la apropiación de las costas por parte de empresas turísticas que construyeron sus instalaciones sobre su línea y la creación de infraestructura portuaria para el arribo de cruceros ha originado un proceso paulatino de privatización de las costas en el sentido de que los establecimientos turísticos localizados sobre la línea de la costa, si bien no violan la zona federal marítima, restringen el acceso a la misma y limitan su uso a sus huéspedes.

Esta situación ha generado una desordenada configuración del espacio, que altera la imagen y fisonomía urbana, y que perjudica la práctica de actividades turísticas y recreativas en el espacio costero.

\section{Antecedentes histórico-geográficos del turismo en Cozumel}

Este apartado revisa los antecedentes históricos a partir de 1950, época de los inicios del turismo en Cozumel. Los principales acontecimientos fueron investigados en fuentes bibliográficas referentes específicamente a Quintana Roo y a Cozumel. También se obtuvieron datos en dos entrevistas realizadas durante el trabajo de campo (2005-2006) al profesor Velio Vivas Valdés, cronista de la ciudad, y al Lic. Gustavo Ortega Joaquín, presidente municipal de Cozumel (2005-2008). 
A partir de la década de los cincuenta, el turismo en Cozumel se vislumbró como la alternativa económica al decaer la actividad portuaria con la crisis chiclera. Hasta este momento, Cozumel constituía una isla "de aventura" a la que llegaban unos pocos visitantes exóticos; los primeros hoteles inaugurados a finales de los años veinte albergaban más contratistas y militares que viajeros.

Entre los acontecimientos que promovieron el turismo destacan las nuevas políticas de desarrollo territorial impulsadas por gobiernos que buscaban la modernización; la visión de pioneros como Chamberlain, quien, desde inicios de los cincuenta, comenzó a establecer contacto con redes turísticas de Estados Unidos y Europa; y la presencia de un reducido grupo de inversionistas locales que entraron al negocio turístico (César y Arnaiz, 1998).

Con respecto al transporte aéreo, el servicio proporcionado por Pan American Airways llegó a Cozumel en 1928. En los primeros años, los aviones acuatizaban en la Laguna Ciega al norte de la ciudad; entre 1930 y 1935 las aeronaves utilizaron la rada (zona costera frente al centro de Cozumel). En esta época, la Compañía Panamericana de Aviación adquirió 160 hectáreas para su campo de aterrizaje localizado a dos kilómetros de San Miguel (Ramos, 1999). Para Vivas, el inicio de la Segunda Guerra Mundial fue lo que favoreció la construcción del actual aeropuerto internacional de Cozumel, dado que, cuando estalló la guerra, el gobierno de Estados Unidos "empieza a investigar la forma de crear un perímetro de protección para resguardar su punto geoestratégico más importante: el Canal de Panamá”. De esta forma, se construyó el actual aeropuerto de Cozumel, inaugurado en 1943, el cual fue realizado por compañías inglesas y estadounidenses con base en criterios estratégicos militares, lo que le otorga una capacidad comercial muy grande.

Los primeros hoteles localizados junto a la playa, en la zona hotelera norte de la isla, según Vivas, fueron el Cabañas del Caribe (hoy denominado Sol Cabañas) construido en 1968, el segundo fue el Playa Azul (1970), y en esta etapa se construye la primera parte del hotel Cozumel Caribe.

En relación con la infraestructura vial, en 1952 se inició un camino terrestre que uniría la localidad con la laguna de Chankanaab. Para este año, el camino llegaba a El Cedral, el poblado más alejado de San Miguel; pero fue hasta 1969, según Vivas, cuando se terminó de construir la carretera costera sur y la transversal, que comunican el núcleo urbano con la costa este de la isla. 
Configuración territorial en las

costas de la isla de Cozumel

A partir de estas obras, también se invirtió a lo largo de la carretera costera sur de la isla. El primer hotel en establecerse en esta área fue el Presidente, inaugurado entre 1969 y 1970. Además, este hotel constituyó el primer alojamiento de cadena con capital internacional establecido en Cozumel.

Una vez construidas las carreteras, se ampliaron las posibilidades de la población local para realizar actividades recreativas en las costas de Cozumel. Hasta ese momento, los sitios costeros utilizados para la recreación eran las playas del centro de la ciudad, la zona llamada la "Quinta" (frente a la Capitanía de Puertos) y Playa San Juan, donde ya se encontraban los hoteles Playa Azul y Cozumel Caribe.Al ser abierta la carretera costera empezó a visitarse la laguna Chankanaab y, con la carretera transversal concluida en 1969, las familias de Cozumel empezaron a dirigirse a la zona oriente de la isla, con lo cual, desde los primeros momentos hasta la actualidad, el sitio preferido ha sido la playa de Chen Río.

Sobre el origen del turismo de buceo en la isla,Vivas señala que se dieron dos circunstancias que la promovieron mundialmente como uno de los destinos de buceo más importantes. La primera fueron las visitas que le hizo Jacques Cousteau, oceanógrafo francés, quien exploró y filmó los arrecifes cozumeleños para presentarlos en su famosa serie de documentales marinos a finales de los años cincuenta e inicios de los sesenta. La segunda circunstancia fue la filmación de la película Un mundo nuevo, la cual dio mucha publicidad nacional e internacional a la isla y a sus arrecifes, porque más de la mitad de la cinta se filmó bajo el agua.

El inicio de la década de los setenta lo marcaron dos significativos acontecimientos para Quintana Roo: por un lado, el comienzo del Plan Maestro de Cancún (1972) elaborado por el Fondo de Promoción e Infraestructura Turística (Infratur) -hoy Fondo Nacional de Fomento al Turismo (Fonatur)-y financiado con créditos del Banco Mundial y del Banco Interamericano de Desarrollo (Instituto de Geografía y Centro de Investigaciones de Quintana Roo, 1980); y, por el otro, el diseño, por parte del gobierno federal, de un Plan de Desarrollo delTerritorio de Quintana Roo, con el fin de fortalecer su proceso de conformación como estado libre y soberano, hecho que se consuma el 8 de octubre de 1974. La nueva entidad nació con los mismos límites y extensión que se le había otorgado al territorio en 1902; se dividía en siete municipios, entre ellos Cozumel, el cual tenía la ciudad cabecera en la isla y una gran porción continental, de $4431.7 \mathrm{~km}^{2}$, que incluía las poblaciones de Playa del Carmen, Tulum, Cobá y Akumal, entre otras (Enciclopedia de Quintana Roo, 1998). 
El Proyecto Cancún contempló, desde su plan maestro, la creación de una ciudad para una población fija de 200000 habitantes, infraestructura en comunicaciones y transporte que incluía un aeropuerto internacional y facilidades de lotes para inversión de equipamiento hotelero, y algunos aspectos más. El caso de Cancún merece especial atención ya que, para 1975, a poco más de un año de haber iniciado su construcción, este centro ya contaba con I 322 cuartos, de los cuales $80 \%$ estaba representado por hoteles de primera y segunda categoría (Instituto de Geografía y Centro de Investigaciones de Quintana Roo, 1980).

A finales de los años setenta, al analizar la cantidad de turistas que visitaron la entidad, se observa una reconfiguración de los flujos turísticos: Cancún se posiciona como el destino más visitado, al superar a Cozumel e Isla Mujeres. Entre 1975 y 1979, Cancún tuvo un incremento de $297 \%$ en sus llegadas, en tanto el aumento de Cozumel para esta misma etapa fue de $69 \%$ y el de Isla Mujeres de sólo 49\% (Instituto de Geografía y Centro de Investigaciones de Quintana Roo, 1980).

En esa época, Cozumel ya tenía consolidada la zona hotelera norte, la cual es anterior al despegue de Cancún. En 1979, la oferta hotelera de la isla de Cozumel era aproximadamente de I 540 cuartos de hotel (Arjona, 2005), menos de la mitad de los que poseía Cancún para ese año.

Durante la década de los setenta, comenzaron a arribar a Cozumel cruceros turísticos; los primeros barcos eran españoles, franceses y rusos; el desembarco de los pasajeros se hacía por medio de barcos tenders. Sin embargo, con el paso de los años las llegadas de cruceros aumentaron, lo cual, sumado a la necesidad de un muelle para el abasto de la población,influyó para la construcción de la primera terminal marítima conocida como "Internacional" (1980). Desde su apertura, se incrementó de forma notoria la afluencia de cruceros. A saber, en 1982 arribaron 3 I 3 navíos, y a finales del decenio, en 1989, fueron 496 los barcos que visitaron Cozumel.

El surgimiento de Cancún favoreció el establecimiento de servicios turísticos a lo largo del eje llamado corredor Tulum-Cancún, lo que se acentuó al modificarse la jurisdicción municipal de Cozumel. El 28 de julio de 1993 se creó el octavo municipio del estado denominado Solidaridad, con parte de la superficie continental de Cozumel, excepto los polígonos en los que se ubican Punta Venado y Xel-Há (Enciclopedia de Quintana Roo, 1998). Este marcó el inicó del desarrollo turístico de la entidad, caracterizado por un uso intensivo 
del suelo costero a lo largo del corredor Tulum-Cancún, conocido hoy como Riviera Maya, al destinar cada vez más tierras a la actividad turística. Esta área ha demostrado ser la zona de mayor crecimiento de la oferta de cuartos del país, con diferentes ritmos pero de forma sostenida en los últimos I 4 años. Entre el 2000 y 2004 hubo un impresionante incremento de 23000 cuartos de hotel, lo cual equivale a un nuevo desarrollo como Ixtapa cada año (Campos, 2004).

A principios de 1990, Cozumel era considerado el destino turístico número dos, después de Cancún, en términos de llegada de turistas. Pero a finales de este decenio, el surgimiento explosivo de la Riviera Maya reconfiguró la geografía turística de la zona. Hoy en día, Cancún es el principal destino turístico del estado, seguido por la Riviera Maya, y por Cozumel en tercer lugar.

Durante los noventa, la isla de Cozumel reorientó sus inversiones turísticas hacia la construcción de dos nuevos muelles en su costa oeste, para el arribo de cruceros turísticos, generando una gran transformación de su litoral. La terminal marítima Puerta Maya, construida entre 1993 y 1996, y Punta Langosta, en I997, aumentaron la capacidad portuaria a seis barcos de forma simultánea.

Además de la infraestructura portuaria, entre 1995 y 2003 se edificaron los grandes hoteles de cadena (resorts) ubicados en la zona sur de la isla frente al Parque Nacional Arrecifes de Cozumel. El proceso de uso turístico de las costas de la isla había empezado con la construcción de las carreteras perimetrales, sin embargo, fue hasta este periodo cuando se dio de forma más intensa y a mayor distancia del centro urbano.

La infraestructura portuaria y el equipamiento hotelero, junto con la aparición de una gran cantidad de clubes de playa, redujeron notablemente las opciones de recreación de la población de Cozumel. Fue entonces cuando playa Casitas empezó a utilizarse como balneario público en respuesta al problema generado por el fuerte crecimiento poblacional y el paralelo aumento de las concesiones de playas. Casitas constituye el último lugar de apropiación de playas por parte de la comunidad local.

\section{Los espacios turísticos y recreativos de las costas de Cozumel}

En esta investigación se clasificaron 65 espacios costeros con uso turístico y recreativo, que, de acuerdo con su ubicación, fueron organizados en cinco sectores territoriales: 
I. Norte: ubicado al septentrión del área urbana de Cozumel.

2. Centro: localizado sobre la avenida Rafael E. Melgar paralelo al malecón, en coincidencia con la longitud urbana costera.

3. Centro-sur: constituido por un continuo de espacios costeros y negocios turísticos de diferentes rubros, emplazado desde el faro de playa Caletita hasta el último muelle de cruceros (Puerta Maya).

4. Sur: ubicado desde el hotel Presidente Intercontinental hasta el Parque Punta Sur.

5. Este: situado en la costa oriental de la isla, desde Punta Molas hasta Punta Celaráin.

La mayoría de los espacios costeros y, por ende, el eje de movilidad de los turistas y visitantes de cruceros, se localiza en la costa oeste de Cozumel, dado que ahí se encuentran la ciudad, los tres muelles de cruceros y los principales negocios turísticos. Esta situación responde básicamente a tres condiciones geográficas: se ubica frente a la Península deYucatán; su costa es poco profunda y menos vulnerable a los vientos provenientes del este; y su cercanía a la barrera de arrecifes, particularmente los espacios que están en el sector sur.

\section{Metodología}

Para clasificar los espacios costeros con uso turístico-recreativo, se establecieron variables de análisis para generar, además de la clasificación, una organización jerárquica de la información obtenida.

La variable básica para definir los tipos de espacios costeros fue la presencia de equipamiento e instalaciones en el lugar, lo cual determinó nueve tipos, ordenados de mayor a menor grado de inversión realizada: resort, hotel, condominio, parque recreativo, hotel con restaurante-bar, club de playa, restaurante-bar, balneario público y playa pública.

A cada uno de éstos se estableció un perfil de usuario, su comportamiento y preferencias; asimismo, fueron estudiadas las características físicas del sitio y la calidad percibida por el usuario.

Para obtener la información necesaria, se realizaron dos periodos de trabajo de campo: el primero en el verano de 2005 y el segundo en las vacaciones 
de Semana Santa de 2006. Este último viaje fue importante para corroborar que, a pesar del daño provocado por el huracán Wilma en la infraestructura portuaria y turística, en la mayoría de los espacios costeros el equipamiento y las instalaciones ya estaban recuperados, o bien, a pesar del deterioro, la gente concurría a ellos.

Puesto que la investigación es cualitativa, las técnicas de recolección de datos también lo son. Los instrumentos aplicados fueron la entrevista semiestructurada, la observación y las entrevistas abiertas a informantes clave.

La entrevista semiestructurada a residentes de la isla de Cozumel fue llevada a cabo en los espacios costeros; se indagó el perfil recreativo de los entrevistados, y su comportamiento y preferencias en el sitio. En total, se efectuaron 48 entrevistas que, según los espacios analizados, se distribuyeron de la siguiente manera: 22 en playas públicas, diez en clubes de playa, seis en restaurantes-bar, cinco en hoteles con restaurante-bar y cinco en el balneario de Cozumel. La información obtenida fue procesada con el programa SPSS (Stadistical Program for Social Sciences) para describir y caracterizar en forma más completa cada subtipo de la tipología.

La observación se aplicó en dos formas: la realizada a los espacios costeros y la efectuada a los visitantes de cruceros. En el primer caso, se evaluó el tipo de costa, las peculiaridades del equipamiento e instalaciones y los aspectos referentes a la calidad general del sitio; en total, se obtuvieron 50 registros. En el segundo, la decisión obedeció al escaso tiempo que permanecen los visitantes en la isla, a la poca disposición a responder encuestas y a que la mayoría de las actividades que llevan a cabo se hacen de forma grupal, coordinadas por un guía local que maneja los tiempos y el acercamiento al grupo. Los aspectos registrados de los visitantes de cruceros en los espacios costeros correspondieron al perfil turístico y a su comportamiento (variables que podían obtenerse a partir de la observación). Esta información fue completada con estadísticas proporcionadas por la Dirección de Turismo Municipal y la Fundación de Parques y Museos de la Isla de Cozumel, que administra el Parque Punta Sur y el Parque Chankanaab, ambos localizados en la costa de la isla y en los que se registra un gran número de visitantes.

Por último, las entrevistas abiertas se aplicaron a diferentes informantes clave según objetivos específicos. Por ejemplo, las realizadas a encargados de establecimientos turísticos costeros, meseros y salvavidas fueron para obtener 
mayor información sobre el comportamiento y las preferencias de los usuarios del sitio; en cambio, las que se les hicieron a autoridades y empleados de la Secretaría de Medio Ambiente y Recursos Naturales (Semarnat) y a la Zona Federal Marítimo Terrestre y Ambientes Costeros (Zofemat) tenían la finalidad de ampliar las características y problemas de estos lugares.

Características territoriales de los espacios costeros con uso turístico-recreativo

Al analizar los principales resultados del trabajo (cuadro I), se observa que el porcentaje mayor corresponde a la playa pública (22\%), la cual no posee ninguna clase de instalaciones o en la que sólo existe la presencia de salvavidas. La mayoría de estos espacios se localiza en el sector centro de la costa oeste y en la costa este, a lo largo de la carretera panorámica.

En segundo lugar destaca el restaurante-bar, con $20 \%$ de los espacios estudiados. Se trata de trece establecimientos de alimentos y bebidas situados en las costas de Cozumel. Esta categoría es la que presenta una distribución territorial más equilibrada, ya que siete se encuentran en la costa oeste y seis en la este.

El tipo hotel, con 17\%, se ubica en tercer lugar. Esta categoría abarca once hoteles pequeños y medianos (el tamaño varía entre 14 y 200 habitaciones), localizados en la siguiente forma: cuatro en el sector norte; tres en el centro junto a la avenida Rafael Melgar; tres más en el sector centro-sur;y, finalmente, un hotel en la costa este de la isla de Cozumel, al margen de la carretera panorámica.

CuAdRo I. CozUmel: CANTIDAD Y TIPOS DE ESPACIOS COSTEROS CLASIFICADOS

\begin{tabular}{lcc}
\hline \multicolumn{1}{c}{ Tipos de espacios } & Cantidad & $\%$ \\
\hline Playa pública & 14 & 21.5 \\
Restaurante-bar & 13 & 20 \\
Hotel & 11 & 17 \\
Club de playa & 8 & 12 \\
Resort & 7 & 11 \\
Condominio & 6 & 9 \\
Hotel con restaurante-bar & 3 & 5 \\
Parque recreativo & 2 & 3 \\
Balneario público & 1 & 1.5 \\
\hline Total & 65 & 100 \\
\hline
\end{tabular}


Configuración territorial en las

costas de la isla de Cozumel

En una primera interpretación puede destacarse que, no obstante el fuerte desarrollo turístico, la creación de infraestructura portuaria y la instalación de empresas de servicios turísticos en las costas de la isla, siguen existiendo espacios disponibles para recreación, cuyo acceso para la población es libre. Sin embargo, al establecer una nueva clasificación de los espacios costeros considerando el tipo de acceso a cada uno de ellos, la lectura de los datos se enriquece con nuevos aspectos (cuadro 2).

Con el criterio del acceso, $40 \%$ de los espacios costeros constituye espacios exclusivos para clientes o huéspedes, de los cuales sobresalen los establecimientos de hospedaje o residenciales turísticos, que dentro de esta categoría suman $92 \%$-hoteles ( $42 \%)$, resorts $(27 \%$ ) y condominios $(23 \%)$; en segundo lugar, los que poseen un acceso limitado por consumo mínimo representan $37 \%$, grupo en el cual los que corresponden a restaurante-bar significan más de la mitad. Por último, con la menor cantidad, se ubican los espacios coste-

CuAdRo 2. CozUmel: ESPACIOS COSTEROS CLASIFICADOS POR TIPO DE ACCESO

\begin{tabular}{|c|c|c|c|c|c|}
\hline $\begin{array}{l}\text { Tipo de } \\
\text { acceso }\end{array}$ & Cantidad & $\%$ & Tipo de espacio & Cantidad & $\%$ \\
\hline \multirow[t]{5}{*}{ Exclusivo } & 26 & 40 & Resort & 7 & 27 \\
\hline & & & Hotel & 11 & 42 \\
\hline & & & Condominio & 6 & 23 \\
\hline & & & Parque recreativo & 2 & 8 \\
\hline & & & Subtotal & 26 & 100 \\
\hline \multirow{4}{*}{$\begin{array}{l}\text { Limitado por } \\
\text { consumo }\end{array}$} & 24 & 37 & Hotel con restaurante-bar & 3 & 13 \\
\hline & & & Club de playa & 8 & 33 \\
\hline & & & Restaurante-bar & 13 & 54 \\
\hline & & & Subtotal & 24 & 100 \\
\hline \multirow[t]{3}{*}{ Público } & 15 & 23 & Balneario público & 1 & 7 \\
\hline & & & Playa pública & 14 & 93 \\
\hline & & & Subtotal & 15 & 100 \\
\hline Total & 65 & 100 & & & \\
\hline
\end{tabular}


ros públicos, con $23 \%$. La mayoría la constituyen las playas públicas y el valor restante es para el único balneario público que tiene baños, palapas, juegos para niños y servicio de salvavidas. Este nuevo análisis evidencia la escasez de espacios costeros públicos para usos turísticos y recreativos, situación que resulta crítica especialmente para los residentes de Cozumel que desean realizar recreación en sus costas.

Al representar en forma cartográfica la clasificación de espacios costeros, se decidió agregar un criterio cuantitativo para diferenciar el tamaño de los sitios (mapa I). La variable cuantificada fue la afluencia diaria de usuarios, dato obtenido mediante una estimación de la cantidad media de turistas, visitantes de cruceros y residentes que concurren al sitio costero, que fue realizada a partir de la observación y de los datos suministrados por los informantes clave en las entrevistas abiertas. El cálculo presentó dificultad pues existe una gran variabilidad en la concurrencia de visitantes por estaciones del año, por temporadas turísticas y por los días de la semana; no obstante, se crearon intervalos de afluencia media y así, con esta información adicional, se enriqueció la clasificación construida.

Por medio de la interpretación cartográfica, se identificaron dos patrones territoriales: uno relacionado con la distribución de los espacios costeros (continuos y dispersos) y el otro referente a la asociación de tipos (heterogéneos y homogéneos).

\section{Patrones de distribución territorial}

Con respecto a la distribución de los espacios costeros se detectaron, según la costa de la isla, dos claras tendencias: una continua y una dispersa.

La distribución continua se visualiza en la costa oeste en cuatro secciones (mapa I):

a) Norte: sector donde, entre 1965 y 1975, se instalaron los primeros hoteles de playa, lo cual, sumado a la cercanía al área urbana y al aeropuerto, justificó este primer continuo. En la actualidad, esta zona es atractiva para desarrollos inmobiliarios residenciales como condominios y country club.

b) Centro y centro-sur: en un primer momento, este continuo se asoció con el muelle del ferry a Playa del Carmen (malecón de la avenida Rafael 
Configuración territorial en las

costas de la isla de Cozumel

354

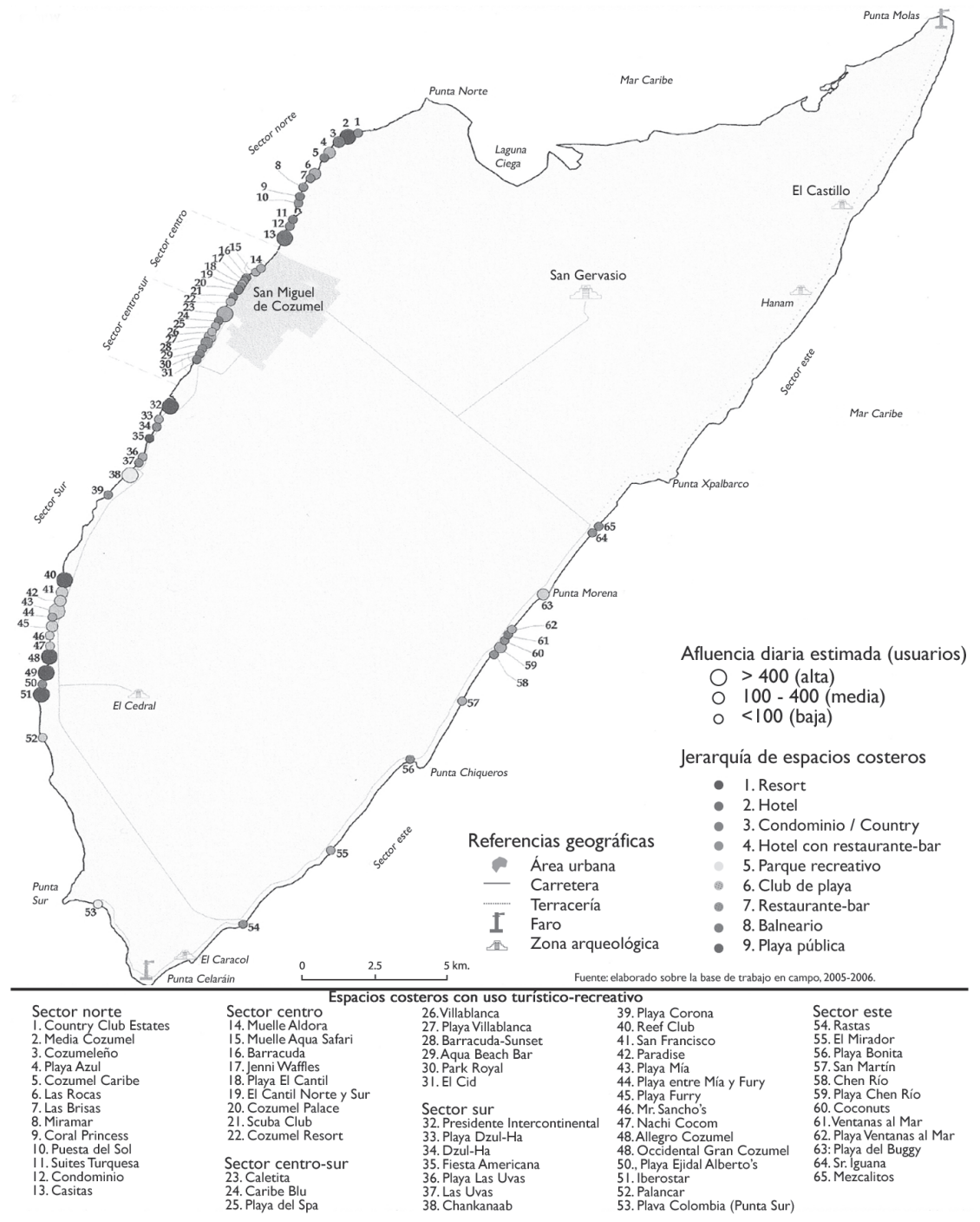

Mapa I. Cozumel, espacios costeros con uso turistico-recreativo, 2006 
Melgar); posteriormente, se extendió por la instalación de los muelles de cruceros en el sector centro-sur hasta Puerta Maya (último de éstos).

c) Sur I: aglutina los espacios costeros instalados a partir de la construcción de la carretera costera sur, en sus primeros kilómetros cerca del Parque Chankanaab.

d) Sur 2: surgió en los años noventa, con la inauguración de clubes de playa y el posterior arribo de empresas internacionales de alojamiento que desarrollaron resorts. En esta sección se localizan las mejores playas y los arrecifes más reconocidos de Cozumel.

La distribución dispersa se observa en la costa este de la isla y surge por las condiciones naturales del área (presencia de dunas e inestabilidad del suelo) que no favorecen la instalación de equipamiento turístico. No obstante, sí llegan a encontrarse algunos espacios costeros a lo largo de la carretera panorámica, ubicados en porciones de costa rocosas como Punta Morena, Chen Río y Punta Chiqueros.

\section{Patrones de asociación tipológica}

La distribución mencionada con anterioridad se presenta entre espacios de asociación heterogéneos y homogéneos (mapa I).

Heterogéneos: se constituyen por espacios asociados a diferentes tipos, en particular de exclusivos o limitados vinculados a espacios públicos (residuales). Las relaciones observadas se dan entre:

- el tipo resort con el tipo club de playa, especialmente en la sección sur 2

- el tipo hotel con el tipo restaurante-bar, en las secciones centro y centro-sur

- el tipo condominio con el tipo playa pública, en las secciones norte y centro

- el tipo club de playa con el tipo playa pública, en la sección sur 2

- el tipo restaurante-bar con el tipo playa pública, en la sección sur I y en la costa este

Homogéneos: se integran por espacios con tipos iguales o similares, se dan únicamente entre el tipo de espacio exclusivo y corresponden a dos categorías 
de equipamiento hotelero: hotel y condominio; están ubicados en el sector norte de la isla, donde inició el desarrollo turístico costero y área que posee una mayor densidad de cuartos (350 por hectárea) (Fonatur y Sectur, 2006).

Con base en el análisis de los patrones territoriales, se observan tres claras tendencias de asociación en la tipología: primero, la originada por un proceso natural de crecimiento del destino turístico y ocupación de espacios costeros por parte de empresas turísticas; segundo, la relacionada con espacios de exclusividad determinados por la lógica de mercado que rige el desarrollo turístico en el norte de Quintana Roo;y tercero, los espacios de carácter residual para los residentes que buscan recreación.

\section{Conclusiones}

Desde el punto de vista turístico, México es reconocido por su gran cantidad de destinos y diversidad de atractivos. Dentro de los que más destacan y que son promocionados internacionalmente se encuentran los de playa. Sin duda, los centros turísticos ubicados en el norte del estado de Quintana Roo son los más importantes de México en función de su capacidad hotelera, la afluencia turística y la captación de divisas.

Con respecto a la configuración territorial del turismo en las costas de Cozumel, los espacios costeros con uso turístico-recreativo investigados están localizados de manera desequilibrada: $81 \%$ se encuentra en la costa oeste, establecido de forma continua en cuatro grandes secciones; $y$ sólo $19 \%$ está ubicado en la costa este de la isla. Esta distribución espacial obedece a que en la primera se encuentran la ciudad y los muelles de cruceros, y que además el sector suroeste de la isla está frente a los arrecifes principales.

Los resultados también revelaron una severa disminución de espacios costeros públicos, en especial playas públicas, a medida que se avanza desde la ciudad sobre el eje norte-sur de la costa oeste. Esta situación se debe a que la zona suroeste de la isla es idónea para la instalación de equipamiento turístico hotelero y clubes de playa, por la cercanía a la costa de los principales arrecifes de Cozumel, la mejor calidad de sus playas y la presencia de espacios relativamente aislados que le otorgan una característica de exclusividad.

En el sector norte de la costa oeste sólo existe un espacio costero público de categoría playa pública, lo cual se explica por la alta densidad de cuartos 
hoteleros y por el aumento de la edificación de condominios. En el corto plazo, es de esperar un mayor número de espacios exclusivos por el incremento de condominios, pues existen al menos tres en construcción en este sector.

De acuerdo con los resultados de la investigación, es posible afirmar que la implantación territorial del turismo en las costas de la isla de Cozumel es producto de la selección preferente de espacios costeros por parte de las empresas turísticas y de desarrolladores inmobiliarios residenciales que responden a los intereses del mercado turístico internacional, representado sobre todo por la demanda estadounidense.

Por último, esta clasificación de espacios costeros con uso turístico-recreativo constituye una expresión espacial del fenómeno turístico actual en Cozumel; pero, en virtud del dinamismo y la diversificación permanente que poseen las prácticas turísticas, puede utilizarse para monitorear y realizar evaluaciones temporales del crecimiento de la oferta del sector en sus costas o llevar a cabo estudios comparativos con otros destinos costeros de México.

\section{FUENTES CONSULTADAS}

Arjona, E. (2005). La satisfacción del turista internacional en la isla de Cozumel. Tesis de licenciatura. México: Universidad de Quintana Roo.

Campos, B. (2004). Procesos de urbanización y turismo en Playa del Carmen, municipio de Solidaridad, Quintana Roo. Disertación doctoral. México: Universidad Nacional Autónoma de México.

César,Alfredo y Stella Maris Arnaiz (1998). Cozumel: los años de espera. México: Fundación de Parques y Museos de Cozumel.

Enciclopedia de Quintana Roo (1998). t. 2. México:Juan Xacur Maíza.

Fonatur y Sectur (2006). Acciones de regeneración y diversificación de atractivos turísticos de Cozumel, Quintana Roo, derivados de los impactos generados por el huracán Wilma. Asistencia técnica a estados y municipios. Información digital suministrada por H.Ayuntamiento de Cozumel.

Instituto de Geografía y Centro de Investigaciones de Quintana Roo (1980). Quintana Roo: organización espacial. Puerto Morelos: Universidad de Quintana Roo.

Ramos, M. (1999). Cozumel. Vida porteña, 1920. México: Universidad de Quintana Roo. 


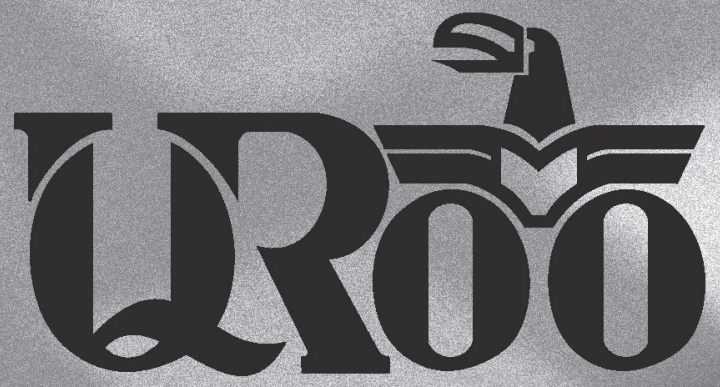

\section{SIG Sistema de Información Geográfica}

El Sistema de Información Geográfica de la Universidad de Quintana Roo ha tenido importantes logros en la adquisición y creación de software y hardware.

Entre las ventajas que podría tener este Sistema de Información Geográfica para una comuna como la de Cozumel figura el manejo del catastro, de la información sobre negocios, de protección civil, de desarrollo urbano, de protección al medioambiente, entre otros grandes rubros. 